\title{
Quantitative global and gene-specific promoter methylation in relation to biological properties of neuroblastomas
}

\author{
Nimrod B Kiss ${ }^{1,3}$, Per Kogner ${ }^{2}$, John Inge Johnsen ${ }^{2}$, Tommy Martinsson ${ }^{4}$, Catharina Larsson ${ }^{1,3^{*}}$ and Janos Geli ${ }^{1,3}$
}

\begin{abstract}
Background: In this study we aimed to quantify tumor suppressor gene (TSG) promoter methylation densities levels in primary neuroblastoma tumors and cell lines. A subset of these TSGs is associated with a CpG island methylator phenotype (CIMP) in other tumor types.

Methods: The study panel consisted of 38 primary tumors, 7 established cell lines and 4 healthy references. Promoter methylation was determined by bisulphate Pyrosequencing for 14 TSGs; and LINE-1 repeat element methylation was used as an indicator of global methylation levels.

Results: Overall mean TSG Z-scores were significantly increased in cases with adverse outcome, but were unrelated to global LINE-1 methylation. CIMP with hypermethylation of three or more gene promoters was observed in 6/38 tumors and 7/7 cell lines. Hypermethylation of one or more TSG (comprising TSGS BLU, CASP8, DCR2, CDH1, RASSF1A and RASSF2) was evident in 30/38 tumors. By contrast only very low levels of promoter methylation were recorded for APC, DAPK1, NORE1A, P14, P16, TP73, PTEN and RARB. Similar involvements of methylation instability were revealed between cell line models and neuroblastoma tumors. Separate analysis of two proposed CASP8 regulatory regions revealed frequent and significant involvement of $\mathrm{CpG}$ sites between exon 4 and 5 , but modest involvement of the exon 1 region.

Conclusions/significance: The results highlight the involvement of TSG methylation instability in neuroblastoma tumors and cell lines using quantitative methods, support the use of DNA methylation analyses as a prognostic tool for this tumor type, and underscore the relevance of developing demethylating therapies for its treatment.
\end{abstract}

Keywords: Neuroblastoma, Pyrosequencing, CIMP, BLU, CASP8, DCR2, CDH1, RASSF1A, RASSF2

\section{Background}

Neuroblastomas and ganglioneuromas are childhood tumors of the sympathetic nervous system that develop from primitive, neural crest-derived cells similar to those of the adrenal medulla and the sympathetic ganglia [1-3]. Afflicted patients present highly variable clinical courses, with spontaneous regression or critical tumor progression as two extreme and contrasting outcomes [2]. A number of clinical features and tumor phenotypes influence the disease outcome; the most important are age at diagnosis, tumor stage, amplification of the $M Y C N$

\footnotetext{
* Correspondence: Catharina.Larsson@ki.se

'Departments of Molecular Medicine and Surgery, Stockholm, Sweden ${ }^{3}$ Center for molecular medicine CMM, Karolinska University Hospital, Stockholm, Sweden

Full list of author information is available at the end of the article
}

oncogene, activating $A L K$ mutations, and somatic loss within chromosomal region $1 \mathrm{p}$ [1,2,4-8]. More recently, hypermethylation of tumor suppressor gene promoters were shown to be frequent in neuroblastoma with possible prognostic implications $[9,10]$.

DNA methylation most commonly refers to methylation of the $\mathrm{C}$ in a CpG dinucleotide motif; a modification of fundamental importance for epigenetic regulation [11]. In normal cells, DNA methylation is central in processes such as expressional regulation, parental imprinting, and $\mathrm{X}$-chromosome inactivation in females [11]. Abundant CpG methylation in promoter regions is widely associated with epigenetic silencing of gene transcription [11]. Aberrant DNA methylation is increasingly observed in various diseases. In cancer, promoter hypermethylation may be an

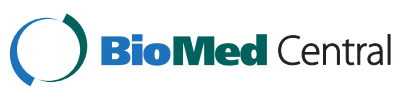


alternative mechanism of tumor suppressor gene (TSG) silencing, which is otherwise associated with genetic mechanisms like mutation and deletion [12-14]. Indeed, concerted anomalous hypermethylation of TSG promoters is reported in an increasing number of cancer types, including among others neuroblastomas [10,15-18], and has been termed $\mathrm{CpG}$ island methylator phenotype (CIMP) [19]. Several reports indicate an association between the CIMP phenotype, advanced tumor disease and adverse outcome [15,16]. However, it is presently unknown whether increasing TSG hypermethylation is selected for during progression [16], or if aberrant DNA hypermethylation triggered by unknown factors confer epigenetic changes responsible for tumor progression.

The present view holds that cancer cells carry localized promoter hypermethylation together with global hypomethylation [20]. The LINE-1 group of retrotransposon elements comprises approximately $17 \%$ of the human genome [21] and has been used as an analogue for genome-wide DNA methylation levels [21-26]. The LINE1 retrotransposon promoter element is frequently hypomethylated in cancers $[24,26]$. The relationship between CIMP and LINE-1 methylation is presently unknown, however a recent study suggested that LINE-1 hypomethylation is inversely correlated with CIMP in colorectal cancer [27].
While several studies have assessed gene methylation in neuroblastomas, the methods employed were nonquantitative $[9,10,28,29]$. The present study was undertaken to quantitatively assess alterations of $\mathrm{CpG}$ methylation globally and in 14 prominent TSG promoters in relation to pathologic phenotypes in neuroblastomas.

\section{Results}

Hypermethylation of BLU, CASP8, DCR2, CDH1, RASSF1A and RASSF2 in neuroblastoma tumors and cell lines

CpG methylation status was assessed for regulatory regions of 14 tumor suppressor genes in 38 tumors and 4 healthy adrenal medullary reference samples (Table 1). For CASP8 two alternative regulatory regions were analyzed, referred to as CASP8 A1 and CASP8 A2, and based on the findings CASP8 A1 was selected for the further analyzes. Several of the investigated TSGs have chromosomal locations that are recurrently lost in neuroblastoma [30,31]: TP73 on 1p, BLU, RARB and RASSF1A on 3p, and PTEN on 10q. Epigenetic silencing of these genes would account for the second hit in Knudson's 2-hit hypothesis. As expected, the majority of genes showed negligible or very low mean levels of methylation (below 10\%) in the reference samples, and the cut-off for hypermethylation was therefore set to $>10 \%$ (Table 1). DCR2, RASSF1A and CASP8A2 displayed higher levels of

Table 1 Overview of methylation levels in normals and primary tumors

\begin{tabular}{|c|c|c|c|c|c|c|c|c|}
\hline & \multirow{2}{*}{$\begin{array}{l}\text { No. of } \\
\text { CpGs }\end{array}$} & \multicolumn{2}{|c|}{ Normals (\% met) } & \multirow{2}{*}{$\begin{array}{c}\text { Hypermethylation } \\
\text { cut-off (\% met) }\end{array}$} & \multicolumn{2}{|c|}{ Tumors (\% met) } & \multicolumn{2}{|c|}{ Tumors > cut-off } \\
\hline & & mean & range & & mean & range & NB & Gang \\
\hline \multicolumn{9}{|c|}{ Genes with detected hypermethylation } \\
\hline$B L U$ & 8 & 4 & $(3-4)$ & $>10$ & 7 & $(2-65)$ & 4 & 0 \\
\hline CASP8 - A1 & 4 & 1 & $(0-3)$ & $>10$ & 16 & $(0-52)$ & 21 & 0 \\
\hline CASP8 - A2 & 5 & 36 & $(24-41)$ & $>50$ & 42 & $(15-64)$ & 9 & 0 \\
\hline$D C R 2$ & 9 & 18 & $(10-21)$ & $>30$ & 12 & $(0-67)$ & 5 & 0 \\
\hline $\mathrm{CDH1}$ & 9 & 4 & $(4-5)$ & $>10$ & 3 & $(1-12)$ & 1 & 0 \\
\hline RASSFIA & 5 & 13 & $(8-21)$ & $>30$ & 43 & $(1-89)$ & 23 & 0 \\
\hline RASSF2 & 6 & 6 & $(4-8)$ & $>10$ & 6 & $(3-11)$ & 1 & 0 \\
\hline \multicolumn{9}{|c|}{ Genes without detected hypermethylation } \\
\hline$A P C$ & 10 & 2 & $(1-4)$ & $>10$ & 1 & $(0-2)$ & 0 & 0 \\
\hline DAPK1 & 13 & 1 & $(0-1)$ & $>10$ & 0 & $(0-1)$ & 0 & 0 \\
\hline NORE1A* & 13 & 1 & $(1-3)$ & $>10$ & 1 & $(0-3)$ & 0 & 0 \\
\hline P14 & 13 & 5 & $(2-11)$ & $>10$ & 1 & $(0-3)$ & 0 & 0 \\
\hline P16 & 4 & 1 & $(0-1)$ & $>10$ & 0 & $(0-1)$ & 0 & 0 \\
\hline TP73 & 19 & 1 & $(1-2)$ & $>10$ & 1 & $(0-2)$ & 0 & 0 \\
\hline PTEN & 10 & 1 & $(1-1)$ & $>10$ & 1 & $(0-1)$ & 0 & 0 \\
\hline$R A R B$ & 10 & 6 & $(5-7)$ & $>10$ & 2 & $(0-5)$ & 0 & 0 \\
\hline \multicolumn{9}{|c|}{ Global methylation } \\
\hline LINE-1 & multiple & 67.7 & $(65.6-70.3)$ & - & 64.1 & (53.9-74.8) & & \\
\hline
\end{tabular}

NB = Neuroblastoma; Gang = Ganglioneuroma

* previously published in [32]. (Geli et al. 2008). 
Table 2 Methylation levels in neuroblastoma cell lines compared to primary tumors

\begin{tabular}{|c|c|c|c|c|c|c|c|c|c|c|c|c|c|c|c|}
\hline $\begin{array}{l}\text { Sample } \\
\text { group }\end{array}$ & $\begin{array}{c}A P C \\
\text { (\% met) }\end{array}$ & $\begin{array}{l}B L U \\
(\%)\end{array}$ & $\begin{array}{l}\text { CASP8- } \\
\text { A1 (\%) }\end{array}$ & $\begin{array}{l}\text { CASP8- } \\
\text { A2 (\%) }\end{array}$ & $\begin{array}{c}D A P K \\
(\%)\end{array}$ & $\begin{array}{c}\text { DCR2 } \\
(\%)\end{array}$ & $\begin{array}{c}\text { CDH1 } \\
(\%)\end{array}$ & $\begin{array}{c}\text { NORE1A } \\
(\%)\end{array}$ & $\begin{array}{l}\text { P14 } \\
(\%)\end{array}$ & $\begin{array}{l}\text { P16 } \\
(\%)\end{array}$ & $\begin{array}{c}\text { TP73 } \\
(\%)\end{array}$ & $\begin{array}{l}\text { PTEN } \\
(\%)\end{array}$ & $\begin{array}{c}\text { RARB } \\
(\%)\end{array}$ & $\begin{array}{c}\text { RASSF1A } \\
\text { (\%) }\end{array}$ & $\begin{array}{c}\text { RASSF2 } \\
\text { (\%) }\end{array}$ \\
\hline \multicolumn{16}{|l|}{ Cut-off } \\
\hline Hypermethylation & $>10$ & $>10$ & $>10$ & $>50$ & $>10$ & $>30$ & $>10$ & $>10$ & $>10$ & $>10$ & $>10$ & $>10$ & $>10$ & $>30$ & $>10$ \\
\hline \multicolumn{16}{|l|}{ NB Cell lines } \\
\hline IMR-32 & 2 & 6 & 77 & 81 & 0 & 80 & 9 & 3 & 5 & 0 & 1 & 1 & - & 93 & 6 \\
\hline SH-5YSY & 1 & 36 & 89 & 85 & 0 & 88 & 19 & 2 & 1 & 1 & 1 & 1 & - & 94 & 11 \\
\hline SK-N-AS & 1 & - & 0 & 77 & 0 & 81 & 6 & 3 & 2 & 0 & 5 & 1 & - & 94 & 26 \\
\hline SK-N-BE & - & - & 94 & - & - & 78 & - & - & - & 1 & - & 0 & - & 95 & - \\
\hline SK-N-DZ & 3 & - & 42 & 39 & 1 & 37 & 12 & 6 & 3 & 1 & 3 & 1 & 4 & 92 & 6 \\
\hline SK-N-F1 & 1 & - & 50 & 72 & 0 & 76 & 10 & 1 & 2 & 1 & 2 & 1 & 8 & 95 & 10 \\
\hline SK-N-SH & 0 & - & 78 & 80 & 0 & 86 & 8 & 2 & - & 1 & 1 & 1 & 3 & 93 & 8 \\
\hline$\overline{\text { All }}$ & 1 & 21 & 61 & 73 & 0 & 75 & 11 & 3 & 3 & 1 & 2 & 1 & 5 & 94 & 11 \\
\hline \multicolumn{16}{|l|}{ NB Tumors } \\
\hline All & 1 & 7 & 16 & 42 & 0 & 12 & 3 & 1 & 1 & 0 & 1 & 1 & 2 & 43 & 6 \\
\hline Number > cut-off & $\mathrm{n}=0$ & $n=4$ & $n=21$ & $n=9$ & $\mathrm{n}=0$ & $n=4$ & $n=1$ & $\mathrm{n}=0$ & $n=0$ & $\mathrm{n}=0$ & $\mathrm{n}=0$ & $n=0$ & $\mathrm{n}=0$ & $n=23$ & $n=1$ \\
\hline
\end{tabular}

met = methylation; Bold indicate hypermethylation.

intrinsic methylation in reference samples, and consequently cut-offs for hypermethylation were set at 30\%, $30 \%$ and $50 \%$ respectively. In the tumor panel, increased promoter methylation was observed in BLU, CASP8, DCR2, CDH1, RASSF1A and RASSF2 (Table 1), possibly providing the second hit for BLU, RARB and RASSF1A. An increase in methylation compared to reference samples was especially prominent in RASSF1A ( $>60 \%$ of the tumors) and CASP8 A1 (> 50\% of the tumors). By contrast hypermethylation was not observed for $A P C, D A P K 1$, NORE1A, P14, P16, TP73, PTEN or RARB (Table 1).

In the seven neuroblastoma cell-lines analyzed hypermethylation was present in BLU, CASP8, DCR2, CDH1, RASSF1A and RASSF2, but was not observed for $A P C$, DAPK1, NORE1A, P14, P16, TP73, PTEN or RARB (Table 2). Hence, the overall methylation pattern in cell lines mirrored that in tumors, i.e. genes that were highly methylated in cell lines also featured hypermethylation in primary tumors (Table 2).

For comparison of methylation densities Z-scores were calculated for each TSG as well as a mean for all TSG analyzed. Taking the whole tumor population into account there was no apparent statistically significant difference between tumors and references with regards to mean Z-scores (Figure 1).

Hypermethylation above the cut-off range was evident for one or more TSG promoters in 30 of the 38 tumors. Additional file 1: Table S1 and Additional file 2: Table S2 details the results for each TSG in the 38 individual tumors concerning mean methylation density of all $\mathrm{CpG}$ sites as well as the range of minimum to maximum values for the individual $\mathrm{CpGs}$ recorded. In all cases genes without detected hypermethylation exhibited mean values below the cut-off at $10 \%$. With one single exception the maximum values recorded for any individual CpG were also below the cut-off at $<10 \%$ (Additional file 2: Table S2). These observations suggest that the APC, DAPK1, NORE1A, P14, P16, TP73, PTEN and RARB promoters are rarely methylated in neuroblastomas. Six genes showed promoter hypermethylation in primary tumors, and in addition, for some samples increased methylation was recorded at individual CpGs although the mean of all CpGs did not reach above the cut-off (Additioanal file 1: Table S1; Figures 2 and 3). BLU exhibited mean methylation $>10 \%$ cut-off in 4 tumors and maximum methylation up to $22 \%$ in 10 additional tumors. Corresponding results were, for CDH1 (1 tumor with mean $>10 \%$ and 1 additional tumor with max at $17 \%$ ), for RASSF2 (1 tumor +24 tumors up to

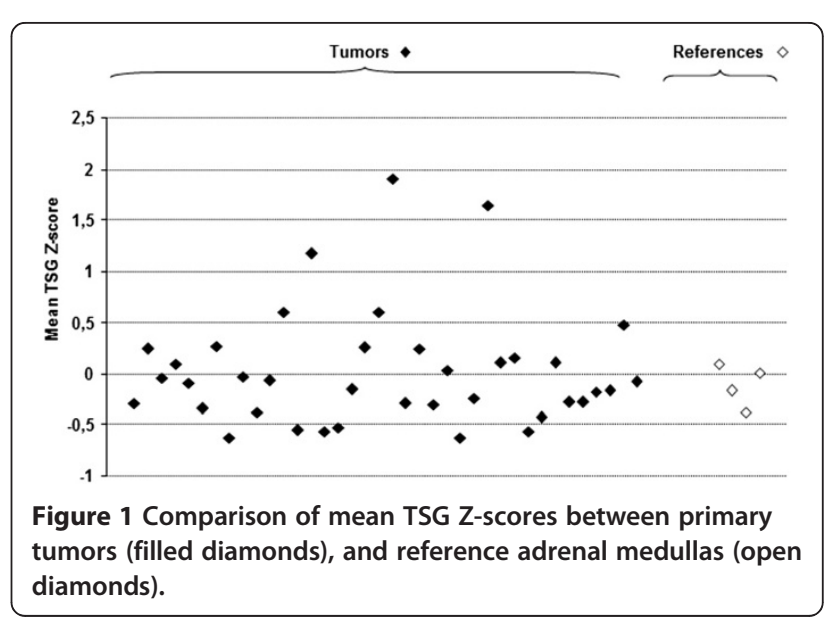




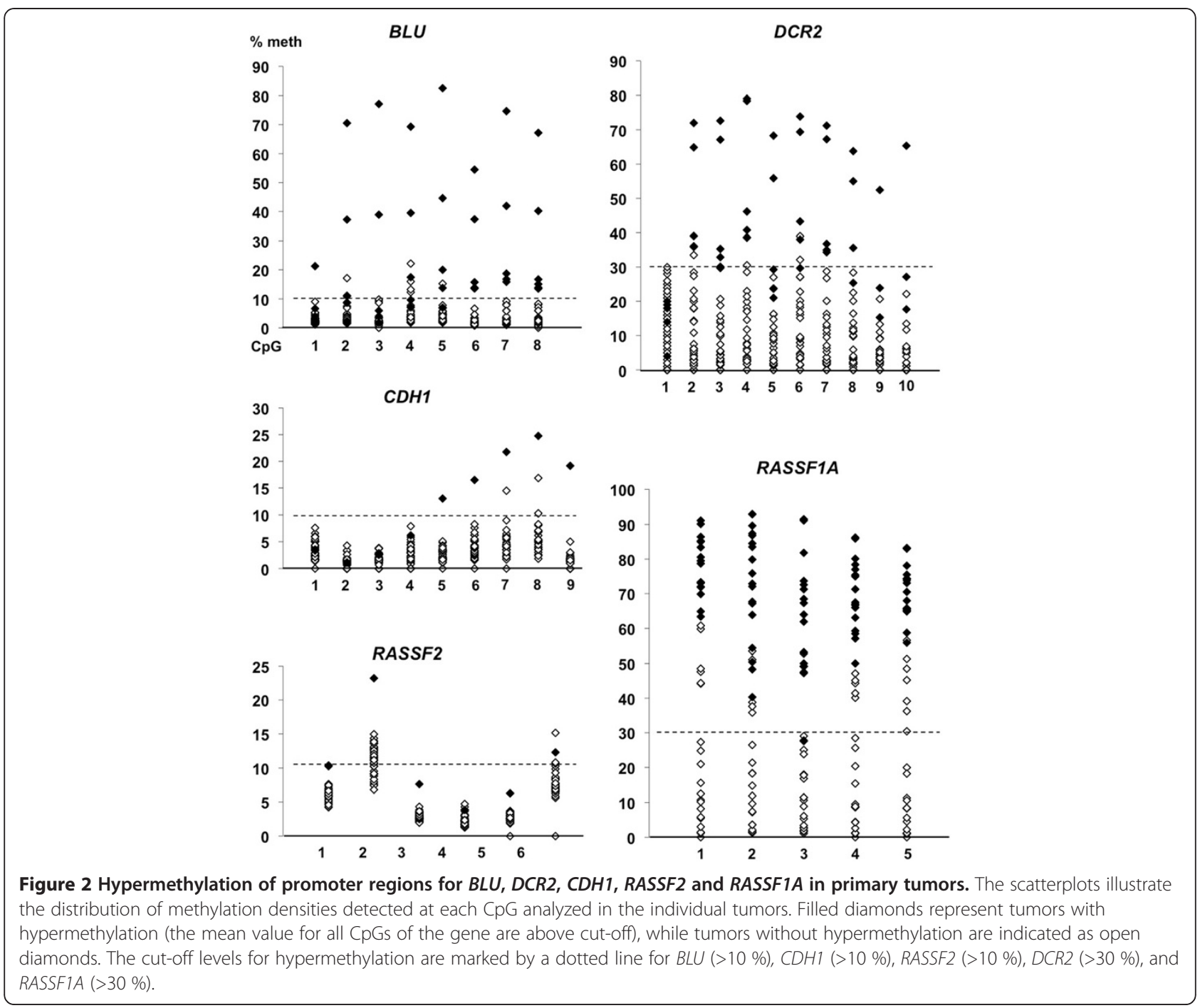

$15 \%$ ), for DCR2 (5 tumors + 3 tumors up to $39 \%$ ), for RASSF1A (23 tumors +1 up to $31 \%$ ), and for CASP8 A1 (21 tumors + 2 tumors up to 21\%). BLU, CDH1, RASSF2, $D C R 2$ and RASSF1A showed quite similar dispersal in methylation densities between individual CpGs (Figure 2). However for CASP8 A1 a gradient of increased methylation was noted from CpG 1 to 2, 3, and 4. (Figure 3). Similarly, CASP8 A2 showed the highest methylation at CPG 2, 3 and 5 , moderate methylation at $\mathrm{CpG} 4$ and the lowest levels at CpG 1 (Figure 3).

\section{CIMP phenotype in neuroblastoma tumors and cell lines}

CIMP was here defined as tumors with concerted hypermethylation in 3 or more of the assessed TSGs in agreement with our previous definitions of this phenotype [32]. Six neuroblastomas, but no ganglioneuromas showed hypermethylation of 3 or 4 genes and thus met the criteria for CIMP (tumors 14, 18, 19, 20, 27 and 35; Table 3). All genes involved in hypermethylation in this study contributed to CIMP, and mean Z-scores and $D C R 2$ Z-scores were significantly higher in tumors classified as CIMP $(\mathrm{p}=<0.003)$. Similarly, all 7 cell-lines carried promoter hypermethylation for 3 to 6 genes in agreement with a CIMP phenotype (Table 2).

\section{Analyses of alternative regulatory regions in CASP8}

The two reported regulatory regions of CASP8 are illustrated in Figure 4, together with the location of primers applied in previous and the present study. In this study, the mean methylation levels for CASP8 A2 were found to be high, between $15-64 \%$, but this was mirrored by prominent methylation also in the reference samples (24-41\%). Nine tumors reached a methylation level above the 50\% cut-off level. In 8 of these 9 tumors hypermethylation was also observed for the CASP8 A1 region (Table 3). For CASP8 A1 methylation levels in reference adrenal medullas were low $<10 \%$, while hypermethylation above the cut-off was recorded in 21 tumors (Table 3). 


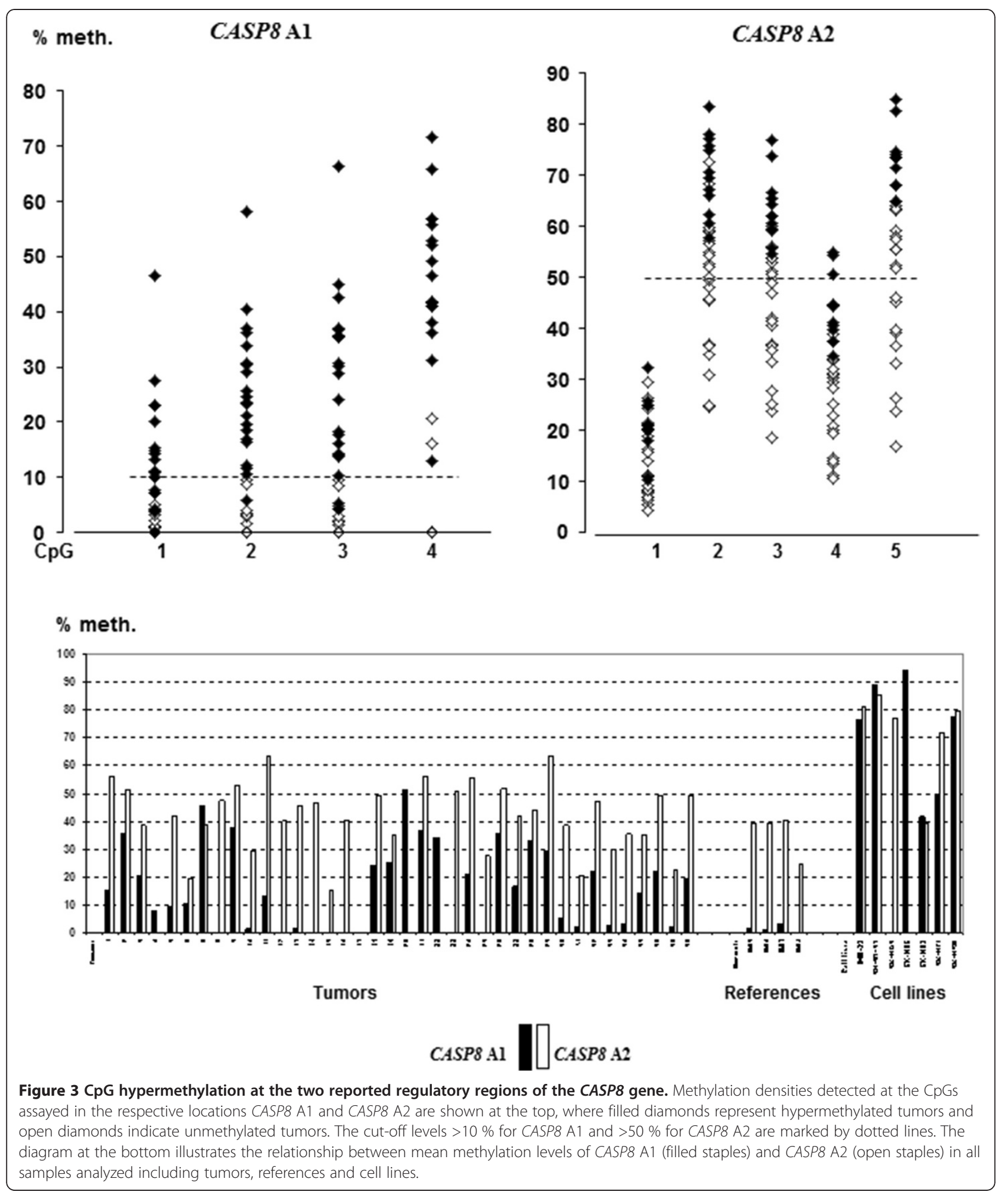

Comparison of TSG methylation with clinical and genetic features

Mean TSG Z-scores were significantly higher in tumors from patients with adverse outcome at follow-up i.e. patients who died of disease (DOD) or were alive with disease (AWD) vs. cases with no evidence of disease (NED) (Kruskal-Wallis $\mathrm{p}=0.04$; Figures 5 and 6). Mean TSG Z-score, CASP8 A1 Z-score and RASSF1A Z-score 
Table 3 Results for dysmethylated promoter regions in primary tumors

\begin{tabular}{|c|c|c|c|c|c|c|c|c|c|c|c|c|c|c|c|c|}
\hline \multirow{2}{*}{$\begin{array}{l}\text { Case } \\
\text { no. }\end{array}$} & \multicolumn{3}{|c|}{ Primary tumor } & \multirow{2}{*}{$\begin{array}{c}\text { High-risk } \\
\text { therapy }\end{array}$} & \multirow{2}{*}{$\begin{array}{c}\text { Follow } \\
\text { up }\end{array}$} & \multirow{2}{*}{$\begin{array}{c}B L U \\
>10 \%\end{array}$} & \multirow{2}{*}{$\begin{array}{c}\text { Casp8 A1 } \\
>10 \%\end{array}$} & \multirow{2}{*}{$\begin{array}{c}\text { CASP8 A2 } \\
>50 \%\end{array}$} & \multirow{2}{*}{$\begin{array}{l}\text { DCR2 } \\
>30 \%\end{array}$} & \multirow{2}{*}{$\begin{array}{l}C D H 1 \\
>10 \%\end{array}$} & \multirow{2}{*}{$\begin{array}{c}\text { RASSF1A } \\
>30 \%\end{array}$} & \multirow{2}{*}{$\begin{array}{c}\text { RASSF } 2 \\
>10 \%\end{array}$} & \multirow{2}{*}{$\begin{array}{c}\text { Total } \\
\text { no. }\end{array}$} & \multirow[t]{2}{*}{ CIMP } & \multirow{2}{*}{$\begin{array}{l}\text { Z-score } \\
\text { all genes }\end{array}$} & \multirow{2}{*}{$\begin{array}{c}\text { LINE-1 } \\
\%\end{array}$} \\
\hline & type & stage & $M Y C N$ & & & & & & & & & & & & & \\
\hline 1 & NB & $4 S$ & - & - & NED & - & CASP8 A1 & + CASP8 A2 & - & - & RASSFIA & - & 2 & - & -0.30 & 64.8 \\
\hline 2 & NB & 1 & - & - & NED & - & CASP8 A1 & + CASP8 A2 & - & - & RASSFIA & - & 2 & - & 0.23 & 66.3 \\
\hline 3 & NB & 3 & - & - & NED & - & CASP8 A1 & - & - & - & RASSF1A & - & 2 & - & -0.04 & 58.0 \\
\hline 4 & NB & 1 & - & - & NED & - & - & - & $D C R 2$ & - & - & - & 1 & - & 0.07 & 65.8 \\
\hline 5 & NB & 4 & yes & yes & DOD & - & - & - & - & - & - & - & - & - & -0.11 & 68.2 \\
\hline 6 & NB & $2 \mathrm{~A}$ & - & - & NED & - & CASP8 A1 & - & - & - & RASSF1A & - & 2 & - & -0.33 & 55.7 \\
\hline 7 & NB & 4 & yes & yes & DOD & - & CASP8 A1 & - & - & - & - & - & 1 & - & 0.26 & 70.0 \\
\hline 8 & NB & 2 & - & - & NED & - & - & - & - & - & RASSFIA & - & 1 & - & -0.65 & 58.2 \\
\hline 9 & NB & 4 & yes & yes & DOD & - & CASP8 A1 & + CASP8 A2 & - & - & - & - & 1 & - & -0.06 & 69.8 \\
\hline 10 & NB & $4 S$ & - & - & NED & - & - & - & - & - & - & - & - & - & -0.37 & 62.9 \\
\hline 11 & NB & 4 & yes & yes & NED & - & CASP8 A1 & + CASP8 A2 & - & - & - & - & 1 & - & -0.06 & 66.4 \\
\hline 12 & NB & 4 & yes & yes & DOD & $B L U$ & - & - & - & - & RASSFIA & - & 2 & - & 0.57 & 62.4 \\
\hline 13 & Gang & - & - & - & NED & - & - & - & - & - & - & - & - & - & -0.55 & 68.9 \\
\hline 14 & NB & 3 & yes & yes & NED & - & - & - & $D C R 2$ & - & RASSFIA & RASSF2 & 3 & CIMP & 1.19 & 57.0 \\
\hline 15 & Gang & - & - & - & NED & - & - & - & - & - & - & - & - & - & -0.58 & 72.6 \\
\hline 16 & NB & 4 & yes & yes & NED & - & - & - & - & - & - & - & - & - & -0.52 & 67.2 \\
\hline 17 & NB & 2 & - & - & NED & - & - & - & - & - & RASSFIA & - & 1 & - & -0.14 & 57.1 \\
\hline 18 & NB & 4 & - & - & NED & - & CASP8 A1 & - & $D C R 2$ & - & RASSFIA & - & 3 & CIMP & 0.24 & 69.9 \\
\hline 19 & NB & 3 & - & - & NED & - & CASP8 A1 & - & $D C R 2$ & - & RASSFIA & - & 3 & CIMP & 0.55 & 59.7 \\
\hline 20 & NB & 2 & - & - & AWD & - & CASP8 A1 & - & $D C R 2$ & $\mathrm{CDH1}$ & RASSFIA & - & 4 & CIMP & 1.87 & 60.2 \\
\hline 21 & NB & 4 & yes & yes & NED & - & CASP8 A1 & + CASP8 A2 & - & - & - & - & 1 & - & -0.30 & 66.8 \\
\hline 22 & NB & 2 & - & - & NED & - & CASP8 A1 & - & - & - & RASSFIA & - & 2 & - & 0.21 & 55.6 \\
\hline 23 & NB & 3 & yes & yes & NED & $B L U$ & - & CASP8 A2 & - & - & - & - & 2 & - & -0.32 & 65.9 \\
\hline 24 & NB & 3 & - & - & NED & - & CASP8 A1 & + CASP8 A2 & - & - & RASSFIA & - & 2 & - & 0.01 & 53.9 \\
\hline 25 & Gang & - & - & - & NED & - & - & - & - & - & - & - & - & - & .0 .62 & 65.9 \\
\hline 26 & NB & 3 & - & - & NED & - & CASP8 A1 & + CASP8 A2 & - & - & - & - & 2 & - & -0.25 & 59.7 \\
\hline 27 & NB & 4 & yes & yes & DOD & $B L U$ & CASP8 A1 & - & - & - & RASSFIA & - & 3 & CIMP & 1.57 & 65.9 \\
\hline 28 & NB & 45 & - & - & NED & - & CASP8 A1 & - & - & - & RASSFIA & - & 2 & - & 0.11 & 58.7 \\
\hline 29 & NB & 3 & - & - & DOC & - & CASP8 A1 & + CASP8 A2 & - & - & RASSF1A & - & 2 & - & 0.14 & 55.0 \\
\hline 30 & NB & 1 & - & - & DOC & - & - & - & - & - & RASSFIA & - & 1 & - & -0.57 & 61.7 \\
\hline 31 & NB & 1 & - & - & NED & - & - & - & - & - & - & - & - & - & -0.43 & 74.8 \\
\hline 32 & NB & 2 & - & - & DOD & - & CASP8 A1 & - & - & - & RASSF1A & - & 2 & - & 0.10 & 64.4 \\
\hline 33 & NB & 1 & - & - & NED & - & - & - & - & - & RASSFIA & - & 1 & - & -0.28 & 67.7 \\
\hline 34 & NB & 4 & - & - & NED & - & - & - & - & - & - & - & - & - & -0.29 & 71.5 \\
\hline 35 & NB & 1 & - & - & NED & $B L U$ & CASP8 A1 & - & - & - & RASSFIA & - & 3 & CIMP & -0.20 & 67.9 \\
\hline 36 & NB & 1 & - & - & NED & - & CASP8 A1 & - & - & - & RASSFIA & - & 2 & - & -0.17 & 66.7 \\
\hline 37 & NB & 3 & yes & yes & NED & - & - & - & - & - & RASSFIA & - & 1 & - & 0.48 & 66.0 \\
\hline 38 & NB & 3 & - & - & NED & - & CASP8 A1 & - & - & - & RASSFIA & - & 2 & - & -0.10 & 67.7 \\
\hline
\end{tabular}

$\mathrm{NB}=$ neuroblastoma; AWD 0 Alive with disease; $\mathrm{DOD}=$ Dead of disease, $\mathrm{NED}=$ No evidence of disease; $\mathrm{DOC}=$ Dead of surgical complications.

were also significantly higher in neuroblastomas than in ganglioneuromas (Mann-Whitney $U$ Test p-values $=0.025$, $0.014,<0.009$ respectively).

As expected significant associations were apparent between tumor stage vs. MYCN amplification; vs. 1p loss; and vs. high-risk, as well as between $M Y C N$ amplification vs. 1p loss and vs. high risk. High-risk was also significantly correlated to poor outcome. The strong correlation between these features support the representativity and relevance of the material under study.

\section{Global LINE-1 methylation}

Methylation density of $L I N E-1$ repeat elements was determined as a measure of the global methylation 

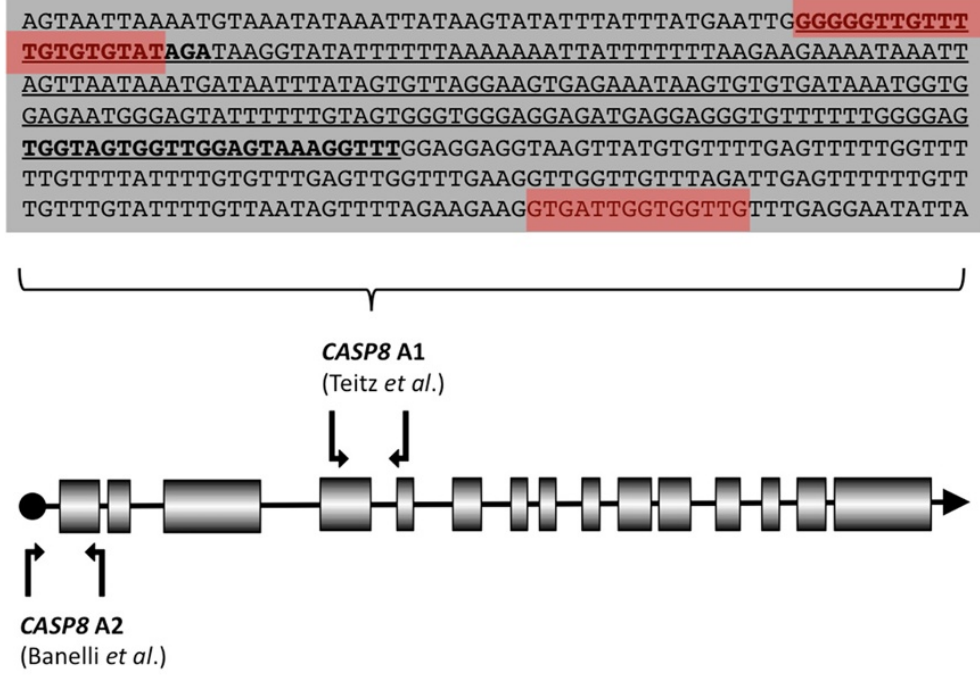

1

TTTTTAAGTTTTGTTGAATTTGTTAGTTAATTTAATAGGAAGTGAGGTTATGGAGGGAGG TAGAAGAGTTAGGGTGGTTATTGAAAGTAAAAGAAATTTTTTTTTGGGAGTTTTTTTTAT TTTTTTTTTTGTTGAGTATGTGGAGTTAGGTAGGTTAGGGGATTTGGAGATTGTGATGGT GTTAGGAAAGGGTGGAGTGGGTGAGTGTTTGTTGTTAAGGTGGTTTTTTTAATAGGAAAT TATAATATTTTTGTTTTTTGATTTGTTTTAGAAATAGGGTTGTGGGGGTGGGGAAGTAAT TTGGATTTGTTTTTTTAGGATATTTTTGGGTGTTGTTTGGTTTAGGTTTTTTGTGTGGT TTTTTTTTGAGTTGTTGTTTTTGATTTTGTTATTTTTTTATTTTGAGTAGTTTTTAGTTT TTTTGTTATTTTTTTGTTTTTTAAGTTTTTTTGTTGTTTTGAATGTAGATATATGGTTTT

Figure 4 Methylation analyzes of the two reported regulatory regions of the CASP8 gene. The location of CASP8 A1 [25] and CASP8 A2 [27] are indicated in the schematic illustration of the CASP8 gene locus. Location of MSP primers applied by Teitz et al., and Banelli et al., are indicated in colour in the sequences shown. The locations of primers used in the present study are marked in bold and the sequence is underlined.

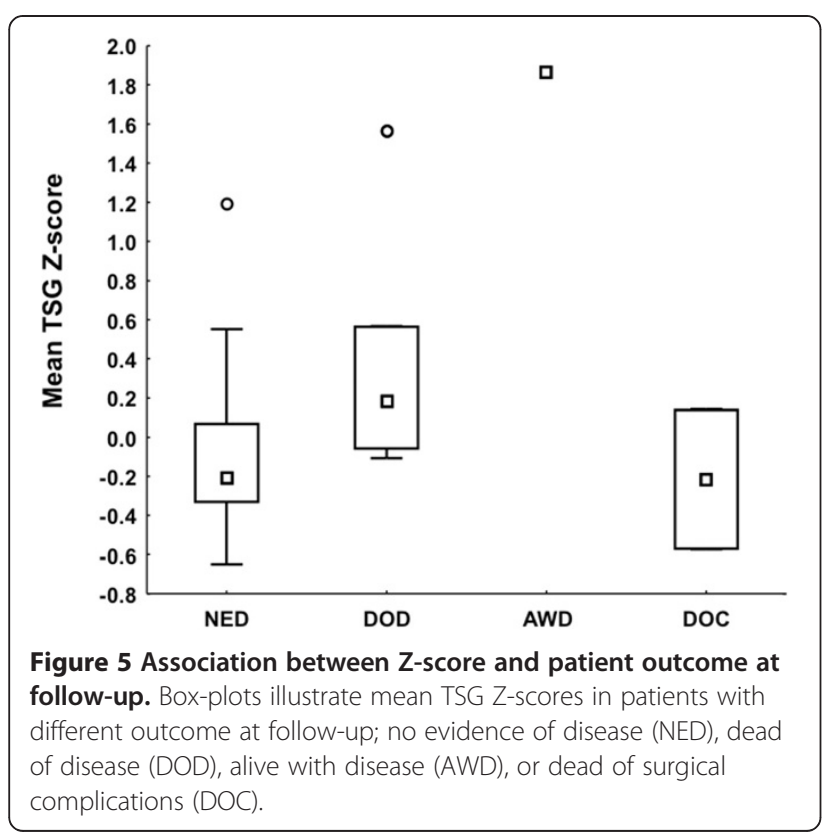

status. High and comparable levels of LINE-1 methylation were recorded in reference samples and primary tumors, while the levels were lower in neuroblastoma cell lines (Figure 7). LINE-1 Z-scores were not significantly different between tumors and reference adrenal medulla. There was a negative correlation between LINE-1 and RASSF1A Z-scores (Spearman Rank Order Correlation -0.56; $\mathrm{p}<0.05)$. Stage 4 neuroblastomas showed higher LINE-1 methylation vs. lower stage tumors (Mann-Whitney $U$ Test; $\mathrm{p}=0.01$; Figure 7). LINE-1 methylation was not correlated to CIMP or other tumor features.

\section{Discussion}

In the current study we used quantitative bisulfite Pyrosequencing to assess promoter methylation levels of tumor suppressor genes known to be frequently hypermethylated in cancer. In 30/38 tumors we found significant hypermethylation in one or more of the following genes; BLU, CASP8, DCR2, CDH1, RASSF1A and RASSF2. Overall Z-scores for the TSGs assessed were significantly associated with adverse outcome. Furthermore, six of the 38 tumors conformed to the criteria for CIMP, i.e. CpG island methylator phenotype. The non- 


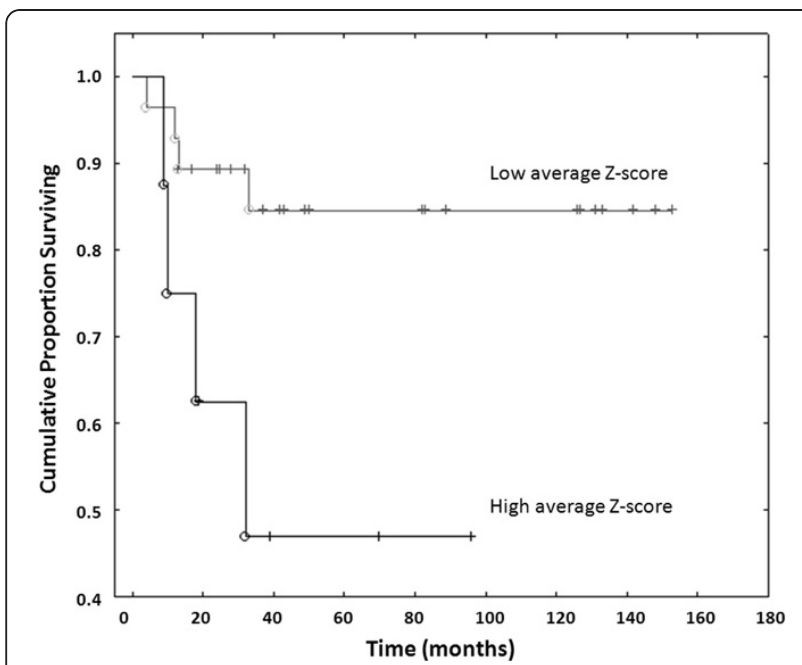

Figure 6 Kaplan-Meier plots illustrating survival in patients with an average Z-score higher or lower than the median of the Z-scores for all methylated TSGs (Table 3). There is a significant difference between the two groups $(p=<0.04)$, indicating adverse outcome in the group with increased mean Z-scores.

tumoral adrenal medullary material in this study represents the closest possible healthy analogue to the cells that comprise neuroblastomas. Neuroblastomas arise solely in neural crest-derived cells of sympathoadrenal lineage ([33] and others), the very cells that would form the adrenal medulla (and abdominal sympathetic ganglia) in healthy individuals [3]. In this current study they were only used to confirm that TSG methylation is low in healthy tissue, and to determine suitable cut-off levels for different TSGs. The level of cut-off for hypermethylation in individual genes was set conservatively to exceed the methylation density observed in the reference adrenal medulla (Table 1). For genes where no methylation was observed the cut-off was set to $10 \%$ to avoid false positives resulting from background fluctuation. For the genes where methylation was detected in reference adrenal medulla (CASP8A2, DCR2, RASSF1A) the cut-off in tumors was set well above the level observed in the reference samples (Table 1). Other recent publications on DNA hypermethylation in neuroblastoma either do not utilize reference controls $[9,10]$, or compare to "normal adrenal" and blood lymphocyte DNA [34]. We used the reference tissue only to assess presence or absence of hypermethylation; for the comparison between methylation levels and clinical/genetic phenotypes, we took into account Z-scores for all 38 tumors in the series, independent of whether they were classified as hypermethylated or not. Hence, methylation levels in reference adrenal medullary material were not included in any statistical calculation.

Although several recent studies have assessed methylation in genes with putative tumor suppressor properties
$[9,10]$, this current study is the first to employ a quantitative method, pyrosequencing, to assess promoter methylation in multiple TSGs. Our data partially corroborate the findings of Grau et al. and Hoebeeck et al., as we find abundant methylation in CASP8A and RASSF1A; however, we did not observe significant hypermethylation in the PTEN promoter as reported by Hoebeeck et al. We here acknowledge the sensitivity of methylation specific PCR (MSP) in detecting low levels of methylation. However, this highly sensitive, nonquantitative assay is known to produce false positive results $[35,36]$. Further, the biological significance of methylation detected by MSP may be limited, as the technique is capable of producing positive results down to a

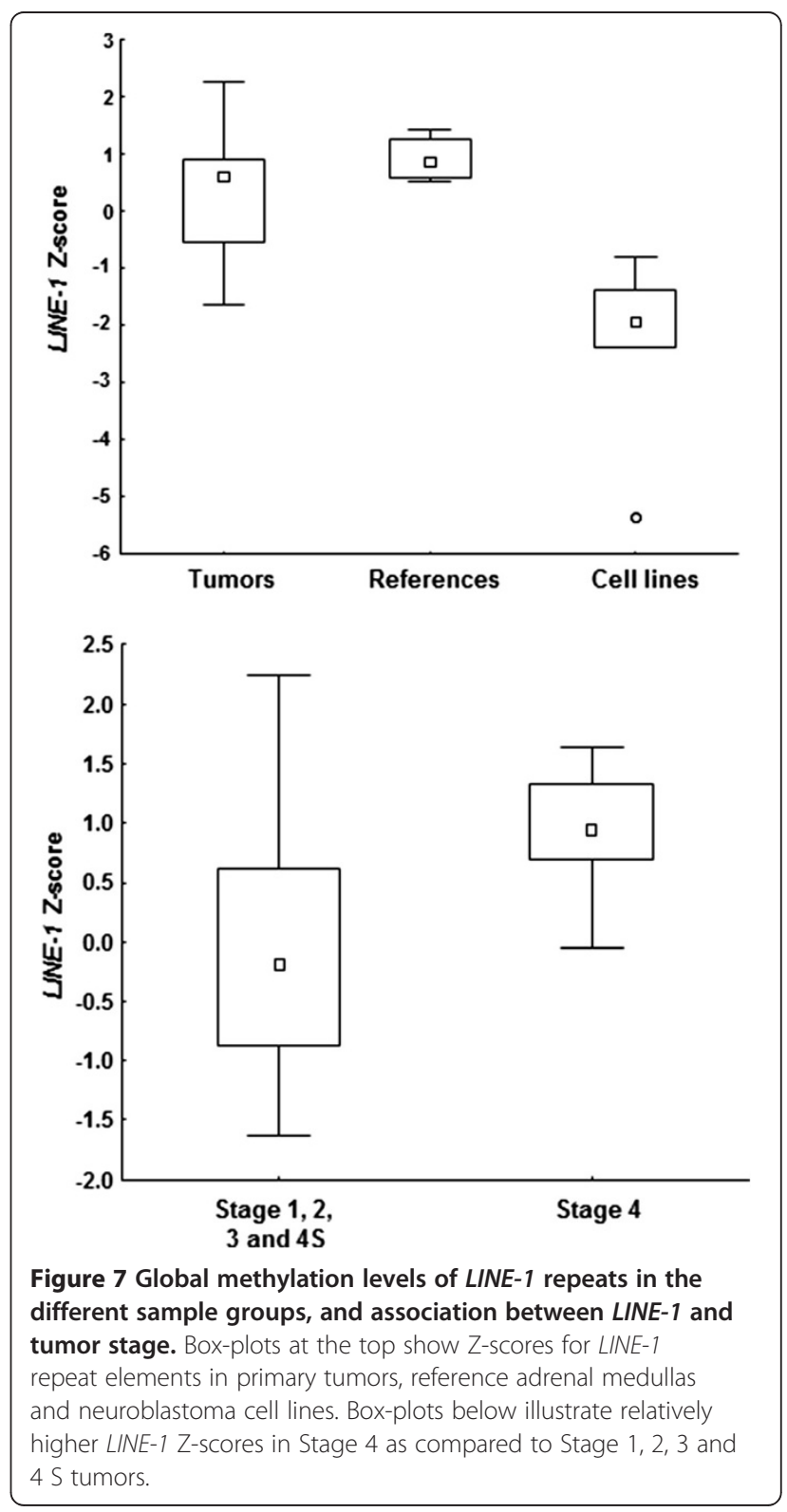


methylation level $<1 \%$ (Rand et al., own observations). This corresponds to a very limited subset of cancerous cells in a tumor, and may in fact represent DNA methylation in contaminating cell types. This underscores the importance of employing quantitative methods when assessing DNA methylation - whenever possible combined with relevant reference samples for the sake of arbitration.

CASP8, defined as a tumor suppressor gene by Teitz et al. [28], promotes apoptosis upon activation of the Fas apoptotic pathway through the Fas ligand [37]. There has been some debate concerning the localization of the CASP8 regulatory region. In 2000 Teitz et al. reported agreement between methylation of a CpG-rich region (defined by GenBank accession number AF210257, positions 536-856; Figure 4) and absent CASP8 expression in neuroblastomas. Also, the region was reported to be methylated almost exclusively in neuroblastomas with $M Y C N$ amplification. Teitz et al. indicated that this could signify that inactivation of the Fas apoptotic pathway is needed for the survival of neuroblastoma cells expressing high levels of $M Y C N$. A contrasting view was presented by Banelli et al. who did not find correlation between CASP8 silencing and MYCN amplification, although higher frequencies of methylation were detected in MYCN-amplified cells [29]. They also argued that the CpG-rich intragenic region assessed by Teitz et al. is not a true regulatory region for CASP8. Instead, a region flanking exon 1 was proposed as the CASP8 promoter (Figure 4). In the current paper we have assessed the regulatory regions suggested in both publications, designated as CASP8 A1 [28] and CASP8 A2 [29] (Figure 4). Furthermore, we have compared the methylation levels in these sites in neuroblastomas to those of healthy reference tissues, which was not undertaken in the previous studies. The differences observed between methylation of the regions CASP8 A1 and CASP8 A2 in reference adrenal medullary DNA are striking: the region CASP8 A1, while abundantly methylated in neuroblastomas (mean methylation 16.2\%; 21 tumors over cut-off), was devoid of methylation in reference adrenal medulla. In contrast, CASP8 A2 had a high degree of methylation in both tumors and references $(9$ tumors over cut-off; mean methylation in tumors $42 \%$ vs. $36 \%$ in reference samples). Thus, hypermethylation at CASP8 A1 was found as a better indicator of a pathologic condition than high methylation at the CASP8 A2 region. Furthermore, CASP8 A1 and CASP8 A2 both showed striking variations in methylation densities between individual CpGs (Figure 3). For CASP8 A1 a gradient with increasing methylation from CpG 1 to CpG 4 was noted, while for CASP8 A2 very high methylation was frequently recorded at CpG 2, 3 and 5 (Figure 3). These observations underline the importance of analyzing more than single $\mathrm{CpGs}$ as an indicator of methylation density.
A long-standing dilemma in neuroblastoma research is the proposed association between CASP8 methylation and MYCN amplification [9,10,28,38]. Like Grau et al. we do not find a correlation between CASP8 A1 methylation and $M Y C N$ amplification [10]. In contrast, Hoebeeck et al., who also assessed the CASP8 A1 region, performed a meta-analysis including a total of 115 neuroblastomas that linked methylation of the CASP8 A1 region with $M Y C N$-amplification [9]. However, the included studies utilized non-quantitative MSP $[9,28,38]$. Our findings provide an epigenetic explanation to a previous study wherein loss of CASP8 protein expression was observed in a majority of neuroblastomas [39]. We further support, backed by quantitative epigenetic data, the observation from that study that no correlation exists between loss of CASP8 and adverse neuroblastoma features and outcomes (such as MYCN amplification and reduced survival) [39].

The CpG Island Methylator Phenotype, CIMP, is characterized by concerted abnormal hypermethylation in CpG rich gene promoters. Such epigenetic remodeling could lead to the simultaneous inactivation of cellular functions that regulate growth, differentiation and apoptosis and thus contribute to neoplasia development and disease phenotype. Indeed, CIMP has been described in a number of cancers, including neuroblastomas, often in conjunction with unfavorable disease progression $[15,16]$. Abe et al. defined CIMP as simultaneous methylation in CpG islands of the $P C D H B$ and $P C D H A$ gene families, and the HLP, DKFZP4511127 and CYP26C1 genes, and found this genotype associated to $M Y C N$ amplification $[17,18]$. In the current study CIMP was observed in 6/38 tumors and in 7/7 cell lines. However, no significant correlations between CIMP and clinical/ genetic features were observed. These results support that concerted promoter hypermethylation is an important facet of the neuroblastoma causality, however if hypermethylation of key TSGs are involved in fatal disease progression they would partly differ from those assessed in this study. By contrast, significantly higher mean TSG Z-scores were observed in tumors with poor outcome at follow-up, which further indicates that hypermethylation is a component of morbidity in neuroblastomas. Analysis of a subset of TSG promoters is likely to render a somewhat fragmental insight into the role of promoter hypermethylation in neuroblastoma development. More global approaches such as methylation arrays are likely to yield a more detailed account of the key genes which, by undergoing hypermethylation, significantly impact tumor progression.

LINE-1 is frequently hypomethylated in cancer [24,26], leading to its activation - which in turn causes genomic instability [40]. In the current study LINE-1 Z-scores 
were higher in stage 4 tumors as compared to tumors of stage $1,2,3$, and $4 \mathrm{~S}$, indicating global hypermethylation. The finding indicates that genomic instability in metastatic neuroblastomas is not caused by LINE-1 activation. Taking into consideration that DNA methylation is a disseminative event, this finding contributes to an emerging picture of aberrant epigenetic patterning in deleterious neuroblastomas.

Several TSGs showed prominent promoter hypermethylation in this study. The two most notably methylated TSG promoters were CASP8 A1 and RASSF1A. Furthermore $B L U$ and $D C R 2$ also exhibited high levels of methylation, and have been reported previously in association with CIMP [17]. Interestingly, serum levels of RASSF1A and DCR2 methylation were reported to have prognostic importance $[41,42]$. The epigenetic inactivation of genes with such diverse tumor suppressive functions as growth arrest (RASSF1A) and apoptosis (CASP8 A1 and $D C R 2$ ) may be requisite for neuroblastoma tumorigenesis. In this regard it is interesting to observe that promoter hypermethylation in the same genes occurred in neuroblastoma cell lines, a phenomenon also observed by Hoebeeck et al. [9]. In the current study the relative methylation patterns were similar between cell lines and primary tumors (Table 2): for TSGs in which high levels of methylation were observed in cell lines, methylation was abundant even amongst the tumor samples. An appealing prospect from this is that neuroblastoma cell lines may be scanned for putative methylation in TSGs, perhaps using array techniques, in order to identify the most relevant TSGs in neuroblastomas. In an approach by Carén et al. [34] that utilized chemical de-methylation of cell lines in combination with expression arrays a number of genes were identified as differentially methylated in neuroblastomas. Interestingly, no bona fide TSGs were identified. Several studies now confirm the presence of gene hypermethylation in neuroblastoma. While this is an important observation the cause-effect relationship between tumorous neuroblastoma and gene hypermethylation should be further investigated to facilitate improved treatment approaches.

\section{Conclusions}

This study provides a quantitative corroboration of previous observations that DNA methylation, in a subset of tumor suppressor genes, is a common event in neuroblastomas; and that it is associated with adverse outcome of the disease. The findings demonstrate a comparable involvement of methylation instability in neuroblastoma tumors and neuroblastoma models, and support an advancement of therapeutic strategies that include the use of demethylating agents to counter TSG silencing in this tumor type.

\section{Materials and methods}

\section{Ethics statement}

The thirty-eight samples of neuroblastoma/ganglioneuroma studied were obtained from patients operated at the Karolinska University Hospital. All samples were initially collected with informed verbal consent from patients or their legal guardians as documented in the patient's medical journal. The collection and subsequent study of the tissue material has been approved by the Karolinska Institutet/Karolinska University Hospital Research Ethics Committee.

\section{Cell lines}

Seven neuroblastoma cell lines were included in the study: SK-N-DZ, SK-N-SH, SK-N-BE, SK-N-FI, SK-NAS, IMR-32, and SH-SY-5Y. Cells were grown as previously described [43].

\section{Patients and tumor samples}

A total of 38 primary tumors from 38 patients were studied (case no 1-38). Clinical details and tumor characteristics have been previously published for all cases [44]. The tumor panel includes 35 neuroblastomas and 3 ganglioneuromas diagnosed between $0 \mathrm{~m}$ and $145 \mathrm{~m}$ of age. Information for $M Y C N$ amplification and $1 p$ loss have been previously reported [44]. Results for NORE1A methylation in tumors have been previously published [44].

\section{Non-tumor controls}

DNA from histopathologically evaluated non-tumoral, healthy adrenal medulla (N1-N4) was acquired from Clinomics Biosciences, Inc. (Watervliet, NY, USA) [45]. The neural crest-derived cells of this healthy adrenal medullary material came from adult individuals, and represents the closest possible analogy to the neural crest-derived cells that comprise neuroblastomas. For the validation of individual pyrosequencing assays methylated human DNA was purchased from Millipore/Chemicon (Billerica, MA, USA) and used as positive control, while normal lymphocyte DNA served as negative control. Serial dilutions were made between in vitro methylated DNA and unmethylated DNA and analyzed by Pyrosequencing to assess potential PCR bias towards either the methylated or the unmethylated form. This was done for all in-house designed Pyrosequencing assays (Additional file 3: Table S3) employing ratios of 100\%, 75\% 50\% 25\% and $0 \%$ of methylated $v$ s. unmethylated DNA.

\section{Extraction of DNA}

Tissues were immediately snap frozen in liquid nitrogen upon surgical removal. DNA extraction from primary tumor samples and neuroblastoma cell lines was carried out either by means of a standard phenol-chloroform 
purification procedure or by applying the ChargeSwitch gDNA Mini Tissue Kit (Invitrogen/Life Technologies Corporation, Carlsbad, CA). A NanoDrop Spectrophotometer (ND-1000) was used to quantify the DNA.

\section{Detection of promoter methylation}

Promoter methylation was quantified by Pyrosequencing for 14 TSGs in tumors, cell-lines, reference samples, and controls. 500 ng of each DNA sample was bisulfite treated using the EZ DNA Methylation Gold Kit (Zymo Research, Orange, CA, USA) and 25-50 ng was subsequently amplified in each gene-specific PCR reaction containing the following reagents: $0.2 \mathrm{mM}$ of each primer (Additional file 3: Table S3), $0.2 \mathrm{mM}$ dNTPs, 1.6 units of HotStarTaq, and 10x PCR buffer (QIAGEN) in a final volume of $50 \mu \mathrm{l}$. The reaction for $D C R 2$ contained additional $\mathrm{MgCl}_{2}$ (QIAGEN) in a final concentration of $3.0 \mathrm{mM}$. PCR conditions were: $95^{\circ} \mathrm{C}$ for 15 minutes then cycled 45 times at $95^{\circ} \mathrm{C}$ for 20 seconds, a gene-specific annealing temperature (Additional file 3: Table S3) for 20 seconds and $72^{\circ} \mathrm{C}$ for 20 seconds, followed by an extension at $72^{\circ} \mathrm{C}$ for 10 minutes. PCR was followed by Pyrosequencing using specific sequencing primers (Additional file 3: Table S3) in either a Biotage $\mathrm{PSQ}^{\mathrm{TM}}$ 96MA Pyrosequencer or in a Biotage PyroMark ${ }^{\mathrm{TM}}$ Q24 Pyrosequencer. Methylation of LINE-1 repeat elements were analyzed as a measurement of global methylation levels using previously described methodology [24]. The standard operating procedure for performing pyrosequencing includes an internal control against incomplete bisulfite conversion: The nucleotide dispensation order is programmed in such a way that $\mathrm{C}$ nucleotides are dispensed where the sequence contains a non-CpG cytosine. If the bisulfite treatment is consummate, all of these Cs have been converted to Ts, and there is no incorporation of the dispensed $\mathrm{C}$ nucleotide. Our assays contained such internal controls, and accordingly full bisulfite conversion was ascertained in all samples.

\section{Statistical analysis}

All calculations were performed in Microsoft Excel 2003 SP3 and in the STATISTICA data analysis software, v. 7.0. $P$ values $\leq 0.05$ were considered significant. Only genes that displayed methylation above the cut-off levels were considered for statistical analysis. To gauge methylation as a continuous variable in multiple promoters, Z-scores were calculated for each gene according to the formula: (Mean of the CpG methylation density of the given promoter for each sample - mean of methylation density for that promoter in the tumor panel)/SD of that methylation density. A mean between Z-scores for all assessed TSG promoters was calculated for each sample, giving an overall methylation score used for comparison between samples. Mean Z-scores for LINE-1 methylation were also calculated. Z-scores and clinical/genetic features (age, stage, high risk therapy, $M Y C N$ amplification, $1 \mathrm{p}$ loss, and outcome) were compared statistically in the STATISTICA 8.0 software (Statsoft Inc., Tulsa, OK, USA). We used Fischer's exact test to compare categorical variables; Mann-Whitney $U$-test and Kruskal-Wallis one-way analysis of variance were used to analyze groups of continuous data; and correlations between continuous data were assessed using Spearman Rank Order correlations. Patients were divided into two groups based on whether their average Z-score was higher or lower than the median of the Z-score average for the methylated genes. The groups were compared by log-rank test; results were illustrated using Kaplan-Meier plots. Two patients with undetermined cause of death were excluded from these calculations.

\section{Additional files}

Additional file 1: Table S1. Methylation densities for hypermethylated genes in primary tumors.

Additional file 2: Table S2. Methylation densities in primary tumors for genes without promoter hypermethylation involvement.

Additional file 3: Table S3. Details of Pyrosequencing assays.

\section{Abbreviations}

DNA: Methylation in neuroblastoma.

\section{Competing interests}

The authors declare that there are no financial or other interests that relate to the current manuscript.

\section{Author's contributions}

NK and JG performed the experiments; NK analyzed the data; PK, JJ and TM provided the clinical material and information; $\mathrm{NK}, \mathrm{CL}$, and $J \mathrm{G}$ designed the study, and NK and CL contributed to writing the manuscript. All authors read and approved the final manuscript.

\section{Acknowledgements}

This study was financially supported by grants from the Swedish Cancer Foundation, the Swedish Research Council, the Swedish Childhood Cancer Foundation, the Göran Gustafsson Foundation for Research in Natural Sciences and Medicine, Karolinska Institutet, and the Stockholm County Council. We are grateful to Stefano Caramuta for the support in constructing the Kaplan-Meier graphs in this publication. The study was supported financially by grants from the Swedish Cancer Foundation, the Swedish Research Council, the Swedish Childhood Cancer Foundation, the Göran Gustafsson Foundation for Research in Natural Sciences and Medicine, Karolinska Institutet, and the Stockholm County Council.

\section{Author details}

'Departments of Molecular Medicine and Surgery, Stockholm, Sweden.

¿Women's and Children's Health, Karolinska Institutet, Stockholm, Sweden. ${ }^{3}$ Center for molecular medicine CMM, Karolinska University Hospital, Stockholm, Sweden. ${ }^{4}$ Department of Biosciences, Sahlgrenska University Hospital, Göteborg, Sweden.

Received: 20 January 2012 Accepted: 10 July 2012

Published: 17 September 2012

\section{References}

1. Brodeur GM: Neuroblastoma: biological insights into a clinical enigma. Nat Rev Cancer 2003, 3(3):203-216. 
2. Schwab M, Westermann F, Hero B, Berthold F: Neuroblastoma: biology and molecular and chromosomal pathology. Lancet Oncol 2003, 4(8):472-480.

3. Larsen WJ, Sherman LS, Potter SS, Scott WJ: Human embryology. London: Churchill Livingstone; 1993.

4. Mossé YP, Laudenslager M, Longo L, Cole KA, Wood A, Attiyeh EF, Laquaglia MJ, Sennett R, Lynch JE, Perri P: Identification of ALK as a major familial neuroblastoma predisposition gene. Nature 2008, 455(7215):930-935.

5. Carén $H$, Abel F, Kogner $P$, Martinsson T: High incidence of DNA mutations and gene amplifications of the ALK gene in advanced sporadic neuroblastoma tumours. Biochem J 2008, 416(2):152-159.

6. Chen Y, Takita J, Choi YL, Kato M, Ohira M, Sanada M, Wang L, Soda M Kikuchi A, Igarashi T: Oncogenic mutations of ALK kinase in neuroblastoma. Nature 2008, 455(7215):971-974.

7. George RE, Sanda T, Hanna M, Fröhling S, Luther W II, Zhang J, Ahn Y, Zhou W, London WB, McGrady P: Activating mutations in ALK provide a therapeutic target in neuroblastoma. Nature 2008, 455(7215):975-978.

8. Janoueix-Lerosey I, Lequin D, Brugières L, Ribeiro A, De Pontual L, Combaret V, Raynal V, Puisieux A, Schleiermacher G, Pierron G: Somatic and germline activating mutations of the ALK kinase receptor in neuroblastoma. Nature 2008, 455(7215):967-970.

9. Hoebeeck J, Michels E, Pattyn F, Combaret V, Vermeulen J, Yigit N, Hoyoux C, Laureys G, Paepe AD, Speleman F: Aberrant methylation of candidate tumor suppressor genes in neuroblastoma. Cancer Lett 2009, 273(2):336-346.

10. Grau E, Martinez F, Orellana C, Canete A, Yañez Y, Oltra S, Noguera R, Hernandez M, Bermúdez JD, Castel V: Hypermethylation of apoptotic genes as independent prognostic factor in neuroblastoma disease. $\mathrm{Mol}$ Carcinog 2011, 3:153-162.

11. Jones PA, Takai D: The role of DNA methylation in mammalian epigenetics. Science 2001, 293(5532):1068.

12. Feinberg AP, Tycko B: The history of cancer epigenetics. Nat Rev Cancer 2004, 4(2):143-153.

13. Esteller M: Epigenetics in cancer. N Engl J Med 2008, 358(11):1148-1159.

14. Baylin SB: Mechanisms underlying epigenetically mediated gene silencing in cancer. Semin Cancer Biol 2002, 12(5):331-337.

15. Teodoridis JM, Hardie C, Brown R: CpG island methylator phenotype (CIMP) in cancer: causes and implications. Cancer Lett 2008, 268(2):177-186.

16. Issa JP: CpG island methylator phenotype in cancer. Nat Rev Cancer 2004, 4(12):988-993.

17. Abe M, Ohira M, Kaneda A, Yagi Y, Yamamoto S, Kitano Y, Takato T, Nakagawara A, Ushijima T: CpG island methylator phenotype is a strong determinant of poor prognosis in neuroblastomas. Cancer Res 2005, 65(3):828.

18. Abe M, Westermann F, Nakagawara A, Takato T, Schwab M, Ushijima T: Marked and independent prognostic significance of the $\mathrm{CpG}$ island methylator phenotype in neuroblastomas. Cancer Lett 2007, 247(2):253-258.

19. Toyota M, Ahuja N, Ohe-Toyota M, Herman JG, Baylin SB, Issa JPJ: CpG island methylator phenotype in colorectal cancer. Proc Natl Acad Sci 1999, 96(15):8681.

20. Feinberg AP, Vogelstein B: Hypomethylation distinguishes genes of some human cancers from their normal counterparts. Nature 1983, 301(5895):89-92

21. Belgnaoui SM, Gosden R, Semmes OJ, Haoudi A: Human LINE-1 retrotransposon induces DNA damage and apoptosis in cancer cells. Cancer Cell Int 2006, 6(1):13

22. Yang AS, Estécio MRH, Doshi K, Kondo Y, Tajara EH, Issa JPJ: A simple method for estimating global DNA methylation using bisulfite PCR of repetitive DNA elements. Nucleic Acids Res 2004, 32(3):e38-e38.

23. Weisenberger DJ, Campan M, Long TI, Kim M, Woods C, Fiala E, Ehrlich M, Laird PW: Analysis of repetitive element DNA methylation by MethyLight. Nucleic Acids Res 2005, 33(21):6823.

24. Hata K, Sakaki Y: Identification of critical CpG sites for repression of L1 transcription by DNA methylation. Gene 1997, 189(2):227-234.

25. Takai D, Yagi Y, Habib N, Sugimura T, Ushijima T: Hypomethylation of LINE1 retrotransposon in human hepatocellular carcinomas, but not in surrounding liver cirrhosis. Jpn J Clin Oncol 2000, 30(7):306-309.
26. Choi IS, Estecio MRH, Nagano Y, Kim DH, White JA, Yao JC, Issa JPJ, Rashid A: Hypomethylation of LINE-1 and Alu in well-differentiated neuroendocrine tumors (pancreatic endocrine tumors and carcinoid tumors). Mod Pathol 2007, 20(7):802-810.

27. Ogino S, Kawasaki T, Nosho K, Ohnishi M, Suemoto Y, Kirkner GJ, Fuchs CS: LINE-1 hypomethylation is inversely associated with microsatellite instability and CpG island methylator phenotype in colorectal cancer. Int J Cancer 2008, 122(12):2767-2773.

28. Teitz T, Wei T, Valentine MB, Vanin EF, Grenet J, Valentine VA, Behm FG, Look AT, Lahti JM, Kidd VJ: Caspase 8 is deleted or silenced preferentially in childhood neuroblastomas with amplification of MYCN. Nat Med 2000, 6(5):529-535.

29. Banelli B, Casciano I, Croce M, Di Vinci A, Gelvi I, Pagnan G, Brignole C, Allemanni G, Ferrini S, Ponzoni M: Expression and methylation of CASP8 in neuroblastoma: identification of a promoter region. Nat Med 2002, 8 (12):1333-1335.

30. Jeison M, Yaniv I, Ash S: Genetic stratification of neuroblastoma for treatment tailoring. Future Oncol 2011, 7(9):1087-1099.

31. Mueller S, Matthay KK: Neuroblastoma: biology and staging. Curr Oncol Rep 2009, 11(6):431-438.

32. Geli J, Kiss N, Karimi M, Lee JJ, Bäckdahl M, Ekström TJ, Larsson C: Global and regional $\mathrm{CpG}$ methylation in pheochromocytomas and abdominal paragangliomas: association to malignant behavior. Clin Cancer Res 2008, 14(9):2551-2559.

33. Nakagawara A: Neural crest development and neuroblastoma: the genetic and biological link. Prog Brain Res 2004, 146:231-242.

34. Carén H, Djos A, Nethander M, Sjöberg RM, Kogner P, Enström C, Nilsson S, Martinsson T: Identification of epigenetically regulated genes that predict patient outcome in neuroblastoma. BMC Cancer 2011, 11(1):66.

35. Aggerholm A, Hokland P, Guldberg P: DAP-kinase CpG island methylation in acute myeloid leukemia: methodology versus biology? Blood 2000, 95(9):2997-2999.

36. Rand K, Qu W, Ho T, Clark SJ, Molloy P: Conversion-specific detection of DNA methylation using real-time polymerase chain reaction (ConLightMSP) to avoid false positives. Methods 2002, 27(2):114-120.

37. Strasser A, Jost PJ, Nagata S: The many roles of FAS receptor signaling in the immune system. Immunity 2009, 30(2):180-192.

38. Gonzalez-Gomez P, Bello M, Lomas J, Arjona D, Alonso M, Aminoso C, Lopez-Marin I, Anselmo N, Sarasa J, Gutierrez M: Aberrant methylation of multiple genes in neuroblastic tumours: relationship with MYCN amplification and allelic status at 1p. Eur J Cancer 2003, 39(10):1478-1485.

39. Fulda S, Poremba C, Berwanger B, Häcker S, Eilers M, Christiansen H, Hero B, Debatin KM: Loss of caspase-8 expression does not correlate with MYCN amplification, aggressive disease, or prognosis in neuroblastoma. Cancer Res 2006, 66(20):10016

40. Symer DE, Connelly C, Szak ST, Caputo EM, Cost GJ, Parmigiani G, Boeke JD: Human L1 retrotransposition is associated with genetic instability in vivo. Cell 2002, 110(3):327-338.

41. Misawa A, Tanaka S, Yagyu S, Tsuchiya K, lehara T, Sugimoto T, Hosoi H: RASSF1A hypermethylation in pretreatment serum DNA of neuroblastoma patients: a prognostic marker. Br J Cancer 2009, 100(2):399-404.

42. Yagyu S, Gotoh T, lehara T, Miyachi M, Katsumi Y, Tsubai-Shimizu S, Kikuchi K, Tamura S, Tsuchiya K, Imamura T: Circulating methylated-DCR2 gene in serum as an indicator of prognosis and therapeutic efficacy in patients with MYCN nonamplified neuroblastoma. Clin Cancer Res 2008, 14(21):7011-7019.

43. Johnsen J, Segerström L, Orrego A, Elfman L, Henriksson M, Kågedal B, Eksborg S, Sveinbjörnsson B, Kogner $P$ : Inhibitors of mammalian target of rapamycin downregulate MYCN protein expression and inhibit neuroblastoma growth in vitro and in vivo. Oncogene 2007, 27(20):2910-2922.

44. Geli J, Kogner P, Lanner F, Natalishvili N, Juhlin C, Kiss N, Clark GJ, Ekström TJ, Farnebo F, Larsson C: Assessment of NORE1A as a putative tumor suppressor in human neuroblastoma. Int J Cancer 2008, 123(2):389-394

45. Geli J, Kiss N, Kogner P, Larsson C: Suppression of RIZ in biologically unfavourable neuroblastomas. Int J Oncol 2010, 37(5):1323-1330.

doi:10.1186/1471-2350-13-83

Cite this article as: Kiss et al:: Quantitative global and gene-specific promoter methylation in relation to biological properties of neuroblastomas. BMC Medical Genetics 2012 13:83. 